(c) 2016. This manuscript version is made available under the Elsevier user license

* Tel: 508-482-2311; Fax: 508-482-3625; E-mail: Fabrice_Gritti@waters.com

\title{
Bridging the gap between gas and liquid chromatography
}

\author{
Fabrice Gritti, Michael Fogwill, Martin Gilar and Joseph A. Jarrell \\ Waters Corporation, Instrument/Core Research/Fundamental \\ Milford, MA, 01757, USA
}

\begin{abstract}
The rapid and complete baseline separation of both volatile $\left(\mathrm{C}_{5}\right.$ to $\mathrm{C}_{16}$ alkanes in gasoline or terpenes in plant extracts) and non-volatile ( $>\mathrm{C}_{20}$ alkanes) organic compounds was achieved by combining 1) low-density fluid chromatography (LDFC) using carbon dioxide at elevated temperature $\left(>90^{\circ} \mathrm{C}\right)$ and low pressure $(1500 \mathrm{psi})$ designed to increase the retention of the most volatile compounds and 2) high-vacuum technology $\left(<10^{-4}\right.$ Torr $)$ in order to preserve the maximum efficiency of short analytical columns $(3.0 \mathrm{~mm} \times 150 \mathrm{~mm}$ packed with $1.8 \mu \mathrm{m}$ fully porous HSS-SB-C 18 particles) when used in LDFC. The volatile compounds are eluted first under isobaric conditions (1500 psi) in less than a minute followed by a linear gradient of the column back pressure (from 1500 to 3500 psi in $5 \mathrm{~min}$ ) for the elution of the non-volatile compounds up to $\mathrm{C}_{40}$. The experimental results demonstrate that LDFC performed with short $3.0 \mathrm{~mm}$ i.d. columns packed with sub-2 $\mu \mathrm{m}$ particles and placed under adiabatic conditions enables the analysts to deliver a single, fast, and high-resolution separation of both volatile and non-volatile compounds.
\end{abstract}

Keywords: Low-density fluid chromatography; supercritical fluid chromatography; vacuum technology; carbon dioxide; volatile and non-volatile compounds; fast and high-resolution separation.

*Corresponding author: (E-mail) Fabrice_Gritti@waters.com; (Tel) 508-482-2311; (Fax) 508-482-3625. 


\section{Contents}

1 Introduction $\quad 3$

2 Experimental $\quad 6$

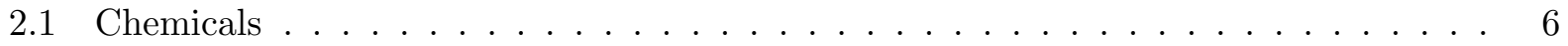

2.2 Instrument and materials $\ldots \ldots \ldots \ldots \ldots \ldots$

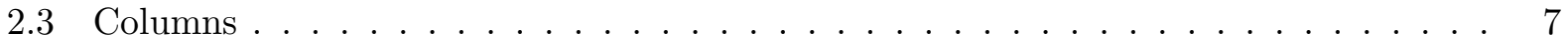

2.4 Chromatographic experiments . . . . . . . . . . . . . . . . . 7

2.4 .1 Volatile terpenes . . . . . . . . . . . . . . . . . 7

2.4 .2 Volatile $n$-alkanes . . . . . . . . . . . . . . . . . 8

2.4.3 Gasoline Gulf $87 \ldots \ldots \ldots \ldots$. . . . . . . . . . . . 8

$2.4 .4 n$-alkanes + paraffin $w a x \ldots \ldots \ldots \ldots \ldots$

3 Results and Discussion $\quad 11$

3.1 Separation of volatiles by isobaric $\mathrm{LDFC} \ldots \ldots \ldots \ldots \ldots$

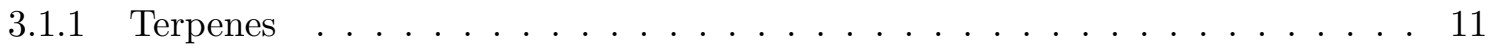

3.1.2 Volatiles $n$-alkanes . . . . . . . . . . . . . . . . . . . . 12

3.1 .3 Gasoline Gulf $87 \ldots \ldots \ldots \ldots \ldots$

3.2 Separation of both volatiles and non-volatile compounds by isobaric and gradient

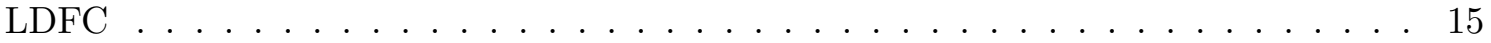

4 Conclusion $\quad 18$

5 Acknowledgements $\quad 19$ 


\section{Introduction}

The high-resolution analysis of volatile compounds present in complex mixtures is successfully carried out by head-space gas chromatography (GC) ${ }^{1}$ while the heaviest fractions containing highmolecular-weight compounds are usually analyzed by high-performance liquid chromatography ${ }^{2}$ or capillary gradient $\mathrm{SFC}^{3}$. The analyst is then constrained to operate different types of instruments and to dispose of a wide spectrum of mobile and stationary phases. This makes the complete analysis of these mixtures relatively complex, costly, and time-consuming. In order to develop more efficient and user-friendly separation methods, the concept of unified chromatography initially relayed by Giddings ${ }^{4}$ half a century ago has slowly emerged into the academic and industrial laboratories with the development of commercial supercritical fluid chromatographs since $1982^{5}$. It was hoped that supercritical fluids (or low-density fluids in general) could enable the analyts to bridge the gap between GC and LC analysis and potentially merge them into a single, faster, more efficient, and more versatile separation technique ${ }^{5}$.

Supercritical fluid chromatography (SFC) is a priori particularly attractive because 1) the viscosity of supercritical fluids (mostly carbon dioxide) is one order of magnitude smaller than that of liquid water, 2) carbon dioxide is an environment-friendly solvent, and 3) the elution strength of supercritical fluids can be easily tuned by independently adjusting its temperature, pressure, and the content of organic solvents ${ }^{6-8}$. Consequently, high-molecular-weight compounds can be easily separated at ambient/moderate temperatures $\left(30-60{ }^{\circ} \mathrm{C}\right)$ by applying either high back column pressures (>2000 psi) or high contents of organic solvent up to $40 \%$ in volume ${ }^{9,10}$. In such conditions, the density of the mobile phase is large and the separation mechanism is similar to that encountered in LC ${ }^{11,12}$ so that retention decreases with increasing temperature. Interestingly, the density of carbon dioxide can also be decreased to such low values ${ }^{11}$ that a reversal in the retention behavior can be observed : retention factors increase with increasing temperature due to the reduction of the intensity of the analyte/fluid interactions. This property of low-density fluids is used to increase the retention of the most volatile compounds: pure carbon dioxide, elevated temperatures $>90^{\circ} \mathrm{C}$, and low column back pressure around 1500 psi can be applied.

At the same time, when using 2.1 to $4.6 \mathrm{~mm}$ i.d. columns, the advantage of low-density fluid 
chromatography (LDFC) can become problematic if the column is not perfectly insulated from the external thermal environment. This is due to the significant amount of heat exchanged between the column and its surroundings as the mobile phase is decompressing ${ }^{13-19}$. In the case of LDFC, the eluent is expanding and cooling during decompression which induces radial density gradients across the column diameter. Capillary columns are not affected by the thermal effects because the volume of mobile phase is extremely small. The impact of thermal effects on chromatographic performance has been observed in SFC for 2.1 to $4.6 \mathrm{~mm}$ i.d. columns ${ }^{20}$ and it has been deeply understood from the simulation of chromatograms under LDFC conditions ${ }^{21-23}$. Peaks are severely distorted, therefore, it is extremely challenging to detect and separate efficiently volatile compounds due to the dramatic loss in column performance: for instance, standard SFC methods cannot provide satisfactory separation of volatile alkanes from $\mathrm{C}_{5}$ (pentane) to $\mathrm{C}_{16}$ because these compounds are too weakly retained and/or they are often co-eluted with the sample solvent ${ }^{3}$. Complex, long, and tedious two-dimensional SFC-GC or GC-GC separation techniques are then required ${ }^{3}$. Therefore, it is relevant to find and apply new strategies that combat against the nefarious effects of thermal phenomena that may affect the efficiency of 2.1 to $4.6 \mathrm{~mm}$ SFC columns. Vacuum ovens that were shown to provide effective solutions to these problems in vHPLC ${ }^{24,25}$ and SFC ${ }^{26,27}$ could then be tested for the analysis of both volatile and non-volatile compounds in real-life sample mixtures.

In this work, it is proposed to separate completely organic volatile compounds $\left(\mathrm{C}_{5}\right.$ to $\mathrm{C}_{16}$ alkanes, terpenes) in a very short time $(<1 \mathrm{~min})$ by one-dimensional LDFC using a $3.0 \mathrm{~mm} \times$ $150 \mathrm{~mm}$ analytical column packed with $2.0 \mu \mathrm{m}$ High Strength Silica (HSS) Stable Bond (SB) $\mathrm{C}_{18}$ fully porous particles. LSFC is used by applying high temperatures $\left(>90^{\circ} \mathrm{C}\right)$ and low column back pressures (1500 psi). Additionally, the peak shape of the retained volatiles is preserved by fully insulating the column in a $3.0 \mathrm{~cm} \times 25 \mathrm{~cm}$ housing chamber at air pressures lower than $10^{-4}$ Torr using a turbomolecular pump ${ }^{24-26}$. The performance of one-dimensional LDFC combined with high-vacuum technologies is compared to that of LDFC under atmospheric pressure and to that of the standard configuration of a SFC system. Finally, it is shown how both volatile and non-volatile compounds can be analyzed and fully separated in a short and single LDFC run by combining 
97 isobaric and back pressure gradient elution modes at high temperatures. Direct applications are 98 given for real sample mixtures such as plant extracts, gasoline, and paraffin wax. 


\section{Experimental}

\subsection{Chemicals}

The mobile phase used was industrial carbon dioxide (99.8\% pure) purchased from Airgas (Worcester, MA, USA). Carbon disulfide was used as the sample solvent: it was HPLC grade from Fisher Scientific (Fair Lawn, NJ, USA). Four sample mixtures were injected: 1) A terpene mixture was prepared by dissolving limonene $(10 \mu \mathrm{L}), \alpha$-humulene $(10 \mu \mathrm{L})$, and $\beta$-cariophyllene $(10 \mu \mathrm{L})$ in $1 \mathrm{~mL}$ of carbon disulfide; 2) A $n$-alkanes mixture was prepared by dissolving $10 \mu \mathrm{L}$ of pentane, hexanes, heptane, 2,2,4-trimethylpentane, octane, decane, undecane, dodecane, tetradecane, hexadecane,

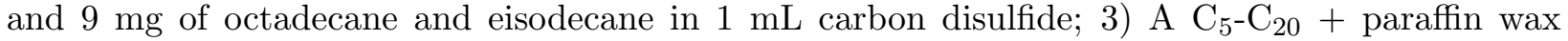
(melting point between 57 and $67{ }^{\circ} \mathrm{C}$, from $\mathrm{C}_{20}$ to $\mathrm{C}_{40}$ ) mixture was prepared by dissolving $25 \mathrm{mg}$ of the paraffin wax in the sample mixture 2); and 4) Gasoline (GULF 87) was directly collected from a local gas station in Milford, MA, USA. Each commercial compound was purchased from Sigma-Aldrich (Suwannee, GA, USA) with a minimum purity of $99 \%$.

\subsection{Instrument and materials}

The ACQUITY UPC ${ }^{2}$ system (Waters, Milford, USA) was used to record the concentration profiles of these analytes. All the parts of the instrument are those of the standard configuration of the ACQUITY UPC ${ }^{2}$ system, except for the $8 \mu \mathrm{L} \mathrm{UV-Vis} \mathrm{detection} \mathrm{cell} \mathrm{which} \mathrm{was} \mathrm{bypassed} \mathrm{and}$ replaced with a flame ionization detector (FID: $\mathrm{H}_{2}+\mathrm{O}_{2}$ ) for detection. A metal tee was used to split the flow of carbon dioxide leaving the column outlet into a $50 \mu \mathrm{m}$ i.d. $50 \mathrm{~cm}$ long capillary (connecting the tee to the FID detector) and a $180 \mu \mathrm{m} \times 40 \mathrm{~cm}$ PEEK tube (connecting the tee to the ABPR unit). The system includes a binary pump with solvent selection valves and a twovalves injection system. A $1 \mu \mathrm{L}$ loop was used with injection volume varying between $0.2 \mu \mathrm{L}$ and $1 \mu \mathrm{L}$. The volume of the eluent mixer is $50 \mu \mathrm{L}$. The ACQUITY UPC $^{2}$ system is controlled by the Empower software 3.0 (Waters, Milford, USA).

The required technical details regarding the materials used or the assembling of the home-made vacuum housing were already reported in previous works ${ }^{24-26}$. Briefly, the vacuum chamber $(25$ 
$\mathrm{cm} \times 6 \mathrm{~cm}$ i.d.) may be pumped by a nominal $60 \mathrm{~L} / \mathrm{s}$ turbomolecular pump (model TMH 071P, 90 $000 \mathrm{rpm}$ ) from Pfeiffer Vacuum (Asslar, Germany). The turbomolecular pump is used to generate pressures as low as $2.010^{-6}$ Torr in about 12 hours. No one vacuum gauge technology is optimal across the full range of air pressure investigated ( $510^{-6}$ Torr to 750 Torr). An inverted magnetron gauge (AIM-C, less than $10^{-3}$ Torr) was used to check the air pressure in the housing chamber and the achievement of a high vacuum $\left(<10^{-4}\right.$ Torr $)$. The gauge was controlled by an Active Gauge Controller. The gauge and the controller were obtained from Edwards High Vacuum (Crawley, $\mathrm{UK})$.

In order to minimize heat losses by radiation, the external surface area of the chromatographic column and most of the inner wall of the vacuum housing were covered with a thin and low emissivity aluminum tape. Aluminum-coated polyester tape was selected and purchased from 3M (Saint Paul, MN, USA). It is generally reported by users that the emissivity coefficient of aluminumcoated surface is about $\epsilon_{A l}=0.04$. For the sake of comparison, the emissivity of machined and polished stainless steel 316 at $297 \mathrm{~K}$ is about $\epsilon_{S S}=0.28$.

\subsection{Columns}

A $3.0 \times 150 \mathrm{~mm}$ column packed with $1.8 \mu \mathrm{m}$ fully porous $\mathrm{HSS} \mathrm{SB}-\mathrm{C}_{18}$ particles from Waters (Milford, MA, USA) was used in this work.

\subsection{Chromatographic experiments}

The chromatographic conditions are described for each sample mixture (terpenes, $n$-alkanes, and paraffin wax).

\subsubsection{Volatile terpenes}

Two different sets of experiment were carried out:

1-Pure carbon dioxide was pumped in a liquid state at $12^{\circ} \mathrm{C}$ at a flow rate of $2.4 \mathrm{~mL} / \mathrm{min}$ and at a steady state pressure of 4323 psi in presence of the chromatographic column. The 
inlet temperature of carbon dioxide was elevated at a temperature of $107^{\circ} \mathrm{C}$ with the mobile phase active preheater. The $8.0 \mathrm{~mm}$ o.d. chromatographic column is encapsulated inside the $6 \mathrm{~cm}$ i.d. housing chamber, which is placed horizontally under still-air conditions at ambient temperature $\left(T=24^{\circ} \mathrm{C}\right)$. The ABPR pressure was set at 1500 psi. $0.2 \mu \mathrm{L}$ of the sample mixture was injected. The housing air pressure was either $1 \mathrm{~atm}$ (vented) or $9.010^{-6}$ Torr (high vacuum).

2- Carbon dioxide was pumped in a liquid state at $12^{\circ} \mathrm{C}$ at a flow rate of $3.0 \mathrm{~mL} / \mathrm{min}$ and at a steady state pressure of 5500 psi in presence of the chromatographic column. The inlet temperature of carbon dioxide was elevated at a temperature of $90{ }^{\circ} \mathrm{C}$ with the mobile phase active preheater. The $8.0 \mathrm{~mm}$ o.d. chromatographic column is either embedded inside the 6 cm i.d. housing chamber, which is placed horizontally under still-air conditions at ambient temperature $\left(T=24{ }^{\circ} \mathrm{C}\right)$, or in the conventional oven compartment of the ACQUITY UPC ${ }^{2}$ instrument. The housing air pressure was $2.010^{-6}$ Torr (high vacuum). The oven temperature is equal to the inlet temperature of carbon dioxide, e.g., $90^{\circ} \mathrm{C}$. The $\mathrm{ABPR}$ pressure was set at 1500 psi. $0.2 \mu \mathrm{L}$ of the sample mixture was injected.

\subsubsection{Volatile $n$-alkanes}

Pure carbon dioxide was pumped in a liquid state at $12^{\circ} \mathrm{C}$ at a flow rate of $3.0 \mathrm{~mL} / \mathrm{min}$ and at a steady state pressure of 5295 psi in presence of the chromatographic column. The inlet temperature of carbon dioxide was elevated at a temperature of $105^{\circ} \mathrm{C}$ with the mobile phase active preheater. The $8.0 \mathrm{~mm}$ o.d. chromatographic column is encapsulated inside the $6 \mathrm{~cm}$ i.d. housing chamber, which is placed horizontally under still-air conditions at ambient temperature $\left(T=24{ }^{\circ} \mathrm{C}\right)$. The $\mathrm{ABPR}$ pressure was set at 1500 psi. $0.3 \mu \mathrm{L}$ of the sample mixture was injected. The housing air pressure was either 1 atm (vented) or $9.510^{-6}$ Torr (high vacuum).

\subsubsection{Gasoline Gulf 87}

Two different sets of experiment were carried out: 
1- Pure carbon dioxide was pumped in a liquid state at $12^{\circ} \mathrm{C}$ at a flow rate of $1.0 \mathrm{~mL} / \mathrm{min}$ and at a steady state pressure of 2565 psi in presence of the chromatographic column. The inlet temperature of carbon dioxide was elevated at a temperature of $105{ }^{\circ} \mathrm{C}$ with the mobile phase active preheater. The $8.0 \mathrm{~mm}$ o.d. chromatographic column is encapsulated inside the $6 \mathrm{~cm}$ i.d. housing chamber, which is placed horizontally under still-air conditions at ambient temperature $\left(T=24{ }^{\circ} \mathrm{C}\right)$. The ABPR pressure was set at $1500 \mathrm{psi} .1 .0 \mu \mathrm{L}$ of the gasoline sample was injected. The housing air pressure was either 1 atm (vented) or $2.510^{-5}$ Torr (high vacuum).

2- Carbon dioxide was pumped in a liquid state at $12^{\circ} \mathrm{C}$ at a flow rate of $1.0 \mathrm{~mL} / \mathrm{min}$ and at a steady state pressure of 2510 psi in presence of the chromatographic column. The inlet temperature of carbon dioxide was elevated at a temperature of $90{ }^{\circ} \mathrm{C}$ with the mobile phase active preheater. The $8.0 \mathrm{~mm}$ o.d. chromatographic column is either embedded inside the 6 cm i.d. housing chamber, which is placed horizontally under still-air conditions at ambient temperature $\left(T=24{ }^{\circ} \mathrm{C}\right)$, or in the conventional oven compartment of the ACQUITY UPC ${ }^{2}$ instrument. The housing air pressure was $2.010^{-6}$ Torr (high vacuum). The oven temperature is equal to the inlet temperature of carbon dioxide, e.g., $90^{\circ} \mathrm{C}$. The $\mathrm{ABPR}$ pressure was set at 1500 psi. $1.0 \mu \mathrm{L}$ of the sample mixture was injected.

\subsection{4 n-alkanes + paraffin wax}

Two different sets of experiment were conducted:

1-Pure carbon dioxide was pumped in a liquid state at $12^{\circ} \mathrm{C}$ at a flow rate of $1.5 \mathrm{~mL} / \mathrm{min}$ and at a steady state pressure of 3210 psi in presence of the chromatographic column. The inlet temperature of carbon dioxide was elevated at a temperature of $70^{\circ} \mathrm{C}$ with the mobile phase active preheater. The $8.0 \mathrm{~mm}$ o.d. chromatographic column is embedded inside the 6 cm i.d. housing chamber, which is placed horizontally under still-air conditions at ambient temperature $\left(T=24^{\circ} \mathrm{C}\right)$. The ABPR pressure was set at 1500 psi during $1.8 \mathrm{~min}$ and was linearly increased to 3500 psi during 5 min. The ABPR pressure was hold at 3500 psi during 
$1 \mathrm{~min}$ and decreased back to $1500 \mathrm{psi}$ in half a minute. $0.2 \mu \mathrm{L}$ of the sample mixture was injected. The housing air pressure was either $1 \mathrm{~atm}$ (vented) or $4.010^{-6}$ Torr (high vacuum). 2-Pure carbon dioxide was pumped in a liquid state at $12^{\circ} \mathrm{C}$ at a flow rate of $1.5 \mathrm{~mL} / \mathrm{min}$ and at a steady state pressure of 3075 psi in presence of the chromatographic column. The inlet temperature of carbon dioxide was elevated at a temperature of $90{ }^{\circ} \mathrm{C}$ with the mobile phase active preheater. The $8.0 \mathrm{~mm}$ o.d. chromatographic column is either embedded inside the 6 cm i.d. housing chamber, which is placed horizontally under still-air conditions at ambient temperature $\left(T=24{ }^{\circ} \mathrm{C}\right)$, or in the conventional oven compartment of the ACQUITY UPC ${ }^{2}$ instrument. The housing air pressure was $2.010^{-6}$ Torr (high vacuum). The oven temperature is equal to the inlet temperature of carbon dioxide, e.g., $90^{\circ} \mathrm{C}$. The $\mathrm{ABPR}$ pressure was set at 1500 psi during 2.0 min and was linearly increased to 3500 psi during 5 min. The ABPR pressure was hold at 3500 psi during 1 min and return to 1500 psi in half a minute. $0.2 \mu \mathrm{L}$ of the sample mixture was injected. 


\section{Results and Discussion}

In the first part of this section, it is demonstrated experimentally that the combination of LDFC (carbon dioxide at elevated temperature and low pressure) with a complete thermal insulation of the chromatographic column (turbomolecular pump, $10^{-5}$ Torr) enables the analyst to enhance the retention of volatile compounds while maintaining the integrity of the peak shape and the highest column efficiencies. Illustrations are given for a mixture of 3 apolar terpenes present in plant extracts, a mixture of 12 alkanes from $\mathrm{C}_{5}$ (pentane) to $\mathrm{C}_{20}$ (eicosane), and a gasoline sample (Gulf 87). In the second part, it is shown how it is possible to merge two separate GC and LC analyses into a single, fast and high-resolution LDFC run for the complete separation of both volatile $\left(\mathrm{C}_{5}-\mathrm{C}_{16}\right)$ and non-volatile (paraffin wax, $\left.\mathrm{C}_{20}-\mathrm{C}_{40}\right)$ compounds.

\subsection{Separation of volatiles by isobaric LDFC}

\subsubsection{Terpenes}

Terpenes are volatile organic compounds that affect the aroma and profile of different strains of cannabis. Those are small molecules such as limonene $(136.2 \mathrm{~g} / \mathrm{mol}), \alpha$-humulene $(204.4 \mathrm{~g} / \mathrm{mol})$, and $\beta$-caryophyllenes $(204.4 \mathrm{~g} / \mathrm{mol}$ ) which are virtually unretained (retention factors are smaller than 0.2 ) on any SFC stationary phase chemistries (diol, $\mathrm{C}_{18}$, ethylpyridine, etc...) when operated under conventional conditions of temperature $\left(50-60^{\circ} \mathrm{C}\right)$ and column back pressure (1800-2200 psi).

Figure 1 shows the two chromatograms recorded at $2.4 \mathrm{~mL} / \mathrm{min}, 107{ }^{\circ} \mathrm{C}$ (preheated mobile phase), and 1500 psi column back pressure (4320 psi inlet pressure) when the column is fully embedded inside the stainless steel air housing chamber (placed under still-air conditions at $24^{\circ} \mathrm{C}$ ) at either atmospheric pressure (top chromatogram) or at a reduced pressure of $10^{-5}$ Torr (bottom chromatogram). Under adiabatic conditions, the retention factors of limonene, $\alpha$-humulene, and $\beta$ caryophyllene are about 1.0, 3.0, and 4.0, respectively. They are noticeably smaller at 0.6, 1.5 , and 2.0 under atmospheric pressure because the column wall may cool down by exchanging heat with the external environment $\left(24^{\circ} \mathrm{C}\right)$. Let recall that the adiabatic decompression of carbon dioxide from 4320 psi to $1500 \mathrm{psi}$ is accompanied by a temperature decrease from $107{ }^{\circ} \mathrm{C}$ to $57{ }^{\circ} \mathrm{C}{ }^{28}$. 
Therefore, when the column is exchanging heat with the surrounding air at $1 \mathrm{~atm}$ and $24^{\circ} \mathrm{C}$, the average density of carbon dioxide along the column is higher than that when the column is thermally insulated under high vacuum. Most remarkably, even though the retention factors are large (1.0 to 4.0) under high vacuum conditions, the quasi-symmetry of the peak shapes is conserved while the same peaks are severely distorted under atmospheric pressure for even smaller retention factors (0.6 to 2.0). This illustrates well the need of combining LDFC with vacuum technology.

Figure 2 compares two chromatograms recorded at a flow rate of $3.0 \mathrm{~mL} / \mathrm{min}, 90^{\circ} \mathrm{C}$, and $1500 \mathrm{psi}$ column back pressure (5500 psi inlet pressure) when the column is either thermally insulated in the vacuum chamber (air pressure $10^{-5}$ Torr, bottom chromatogram) or placed in the conventional oven compartment of the ACQUITY UPC ${ }^{2}$ instrument (top chromatogram). Note that, in comparison to Figure 1, the elution times are comparable for both thermal configurations (retention factors of 0.51 .4 , and 1.8) because the air in the oven compartment is now heated at $90^{\circ} \mathrm{C}$ instead of ambient temperature. The differences between the inlet and outlet temperatures are then comparable (inlet: $90^{\circ} \mathrm{C}$; outlet: $49^{\circ} \mathrm{C}$ ) as the pressure of carbon dioxide is dropping from 5500 psi to 1500 psi. However, even though the retention factors are identical, the conventional instruments cannot maintain the column efficiency at a high level with low-density carbon dioxide. The column has to be completely thermally insulated in order to deliver high-resolution performance for volatile compounds.

\subsubsection{Volatiles $n$-alkanes}

Volatile $n$-alkanes from $\mathrm{C}_{5}$ to $\mathrm{C}_{20}$ can be easily separated by capillary GC (5 m long, $0.5 \mathrm{~mm}$ i.d., $0.2 \mu \mathrm{m}$ film thickness) using helium as the carrier gas and by applying a temperature gradient from $-30^{\circ} \mathrm{C}$ to about $200{ }^{\circ} \mathrm{C}{ }^{3}$. Yet, the analysis times are relatively long around $10-20 \mathrm{~min}$ and the most volatile compounds $\left(\mathrm{C}_{5}\right.$ to $\left.\mathrm{C}_{8}\right)$ co-elute with the sample solvent. The advantage of capillary GC is that thermal effects are not present, so, the peak shapes are always excellent under linear conditions (no sample volume or concentration overload).

In this work, it is attempted to reduce the analysis time by using a short $3.0 \mathrm{~mm} \times 15 \mathrm{~cm}$ analytical columns packed with fully porous materials. The advantage of columns packed with fully porous particles with respect to capillary columns is their very large surface-to-volume ratio. 
This ratio can be calculated from the packing density (typically $\rho_{p}=0.7 \mathrm{~g} / \mathrm{cm}^{3}$ ), the specific surface area of the particles (around $S_{p}=200 \mathrm{~m}^{2} / \mathrm{g}$ ), and the total column porosity $\left(\epsilon_{t}=65 \%\right.$ is a standard value). The surface-to-volume ratio is then equal to $\frac{\rho_{p} S_{p}}{\epsilon_{t}}=210^{8} \mathrm{~m}^{-1}$ for packed columns. The same surface-to-volume ratio is $\frac{2}{r_{c}}$ for a capillary column where $r_{c}$ is the inner radius of the capillary. Therefore, taking a $0.5 \mathrm{~mm}$ i.d. capillary, the same phase ratio is equal to only $810^{3} \mathrm{~m}^{-1}$, four to five orders of magnitude smaller than that of a packed column. Additionally, the retention of the most volatile compounds are increased by using low-density carbon dioxide at $105{ }^{\circ} \mathrm{C}$ and for a low column back pressure of only $1500 \mathrm{psi}$. The flow rate was set at $3.0 \mathrm{~mL} / \mathrm{min}$ (inlet pressure $5295 \mathrm{psi}$ ) in order to maximize speed. The sole disadvantage of a $3.0 \mathrm{~mm}$ i.d. columns with respect to a $0.5 \mathrm{~mm}$ i.d. capillary column is the large amount of heat absorbed by carbon dioxide as it decompresses and the subsequent severe deformation of the peak shape ${ }^{20}$. Nevertheless, this problem can alleviated by thermally insulating the column under high vacuum ${ }^{24,25}$ as was observed for the terpenes.

Figure 3 shows the same chromatograms as in Figure 1, except for the mixture of 12 volatile $n$-alkanes. Again, as expected, the retention times observed for a housing air pressure of 1 atm are smaller than those measured when the column is thermally insulated. It is because the difference between the inlet and outlet temperatures increases when the column is allowed to exchange heat with the cool ambient air at $24^{\circ} \mathrm{C}$. Under adiabatic conditions, the outlet temperature is expected to be $54{ }^{\circ} \mathrm{C}$. Again, it is remarkable that the complete baseline resolution of all the $n$-alkanes is achieved in less than a minute in isobaric LDFC while it would take over $10 \mathrm{~min}$ in temperature gradient GC. In contrast, under standard conditions (1 atm housing air pressure), it is not possible to fully resolve the $\mathrm{C}_{5}$ to $\mathrm{C}_{8}$ fraction. Again, this example illustrates perfectly why vacuum technology is necessary in LDFC in order to observe good peak shapes and to reach high-resolution levels.

\subsubsection{Gasoline Gulf 87}

Figure 4 shows the recorded chromatograms of $1 \mu \mathrm{L}$ of commercial gasoline (Gulf 87) recorded at $1.0 \mathrm{~mL} / \mathrm{min}, 105{ }^{\circ} \mathrm{C}$ preheated carbon dioxide, and at 1500 psi ABPR pressure (inlet pressure $2560 \mathrm{psi}$ ) when the column is immersed in the housing chamber at either atmospheric pressure 
(top chromatogram) or $10^{-5}$ Torr (adiabatic conditions, bottom chromatogram). Under adiabatic conditions, the outlet temperature is expected to be $72{ }^{\circ} \mathrm{C}{ }^{28}$. As for the previous $n$-alkane sample mixture, the retention factor are clearly smaller when the housing chamber is vented enabling heat exchanges between the column wall and the cool ambient air at $24{ }^{\circ} \mathrm{C}$. In contrast, it is interesting to observe that the retention time of ethanol $(10 \%$ is present in commercial gasoline, overloaded band eluted after $8 \mathrm{~min}$ ) is increasing as the average density of carbon dioxide is increasing along the column: the retention of ethanol onto the non-endcapped HSS-SB- $\mathrm{C}_{18}$ column is not driven by the solubility parameter of carbon dioxide but by the adsorption energy onto the active and accessible surface silanols at the surface of the stationary phase (classical van't Hoff temperature behavior). Again, the application of a high vacuum in the housing tube enable the analyts to observe a larger number of peaks than those detected when the column is not thermally insulated.

Figure 5 shows the same chromatograms as in Figure 4, except the preheated mobile phase temperature was $90^{\circ} \mathrm{C}$ (adiabatic conditions, bottom chromatogram) and the column was placed in the oven compartment of the ACQUITY $\mathrm{UPC}^{2}$ instrument (atmospheric pressure, $90^{\circ} \mathrm{C}$ oven, top chromatogram). Under adiabatic conditions, the outlet temperature is expected to be $60{ }^{\circ} \mathrm{C}{ }^{28}$. As for the terpenes (see Figure 2), the retention times are now nearly the same. In fact, when the column is placed in the oven compartment, they are necessarily slightly larger than those observed when the column remains in the vacuum chamber: under the influence of the heated oven at $90{ }^{\circ} \mathrm{C}$, the outlet temperature is inevitably larger than $60^{\circ} \mathrm{C}$. This is confirmed by the smaller retention time of ethanol when the column is placed in the oven. In the end, under steady-state regime, the temperature difference between the oven and the column is not extreme, so, the resolution level remains acceptable under conventional thermal configuration (top chromatogram). For that all, the chromatographic resolution is visibly better when the column is fully insulating (bottom chromatogram).

Note that the severe peak tailing observed for ethanol is of thermodynamic origin. It is caused by the very large equilibrium constant of ethanol between low density carbon dioxide and scarce active adsorption sites present at the surface of the nonendcapped HSS-SB-C ${ }_{18}$ stationary phase. This peak tailing was also observed for 
various polar organic modifiers such as acetonitrile, methanol, and 2-propanol ${ }^{27}$. Isolated but still accessible residual silanols could definitely be involved and responsible for this observation. In this particular application, this situation was beneficial because the wide FID signal of ethanol did not overlap with those of the apolar compounds. Yet, one simple way to cope with the retained and wide peaks of ethanol is to add a very small amount of polar organic modifier $(<1 \%, v / v)$ in the mobile phase in order to trap the active sites.

\subsection{Separation of both volatiles and non-volatile compounds by isobaric and gradient LDFC}

The separation of both volatiles and non-volatiles usually requires two separate analyses, one by GC, the other by LC. High temperature capillary SFC using a pressure gradient has been also used ${ }^{3}$, however, it is extremely challenging to retain the most volatile compounds $\left(<\mathrm{C}_{20}\right)$ and the analysis times are relatively long (> $10 \mathrm{~min})$. Therefore, we propose here to apply LDFC using short $3.0 \mathrm{~mm}$ i.d. columns packed with sub-2 $\mu \mathrm{m}$ particles under adiabatic conditions in order to provide faster separations while maintaining high resolution levels.

Figure 6 compares the chromatograms of a mixture of volatile $n$-alkanes $\left(\mathrm{C}_{5}-\mathrm{C}_{20}\right)$ and nonvolatile (paraffin wax, $\mathrm{C}_{20}-\mathrm{C}_{40}$ ) compounds by applying a constant flow rate of $1.5 \mathrm{~mL} / \mathrm{min}$, a preheated mobile phase temperature of $65^{\circ} \mathrm{C}$, and an initial ABPR pressure of 1500 psi (the inlet pressure was measured at $3460 \mathrm{psi}$ ). For this application, these isobaric conditions are used to elute the volatile compounds during $1.8 \mathrm{~min}$, then, a linear gradient of ABPR pressure (from 1500 to $3500 \mathrm{psi}$ ) was applied during $5 \mathrm{~min}$ for the elution of the heaviest alkanes. The column is maintained in the vacuum chamber at either atmospheric pressure (top chromatogram) or at $10^{-5}$ Torr (adiabatic conditions, bottom chromatogram). Under adiabatic conditions, the outlet temperature is expected to be close to $43{ }^{\circ} \mathrm{C}{ }^{28}$. Remarkably, a better peak resolution is observed for both volatile and non-volatile compounds when the column is not allowed to exchange heat with the cool external environment (ambient air at $24^{\circ} \mathrm{C}$ ).

However, it appears from the bottom chromatogram in Figure 6 that the peak shapes of the 
heaviest components from the paraffin wax are not as thin and symmetrical as those observed for the volatile compounds during isobaric conditions. It is important to recall that the ABPR gradient necessarily induces a change for the steady-state temperature regime of the column: initially, before the ABPR gradient starts and under adiabatic conditions, the temperature of carbon dioxide decreases from $65^{\circ} \mathrm{C}$ at the column inlet to $43{ }^{\circ} \mathrm{C}$ at the column outlet. Assuming that the end of the ABPR gradient (3500 psi) would be held indefinitely, the temperature of carbon dioxide would then decrease from the same inlet temperature of $65^{\circ} \mathrm{C}$ to $59^{\circ} \mathrm{C}{ }^{28}$. So, the wall temperature tends to heat up during the ABPR gradient creating a heat flux from the bed immersed in carbon dioxide to the stainless steel wall. A transient thermal regime is taking place, heat is transferred from the continuously warming carbon dioxide (from $43{ }^{\circ} \mathrm{C}$ to $59{ }^{\circ} \mathrm{C}$ at the column outlet) to the stainless steel wall, radial density gradients are temporarily formed across the bed diameter, and the peaks recorded during this transient regime are inevitably distorted. The fundamental problem is that the chromatographic bed immersed in the mobile phase is not thermally insulated from the surrounding stainless steel walls. Because a fluid (carbon dioxide) flowing in a metal tube (stainless steel) is an excellent heat exchanger (its heat exchange coefficient is one to two orders of magnitude more intense than that of a gas flowing on a tube), it becomes critical to minimize the temperature difference of the flowing carbon dioxide before and after the ABPR pressure gradient.

Accordingly, the complete thermal insulation of the column is not the proper thermal configuration during $\mathrm{ABPR}$ gradients because the amplitude of the temperature change between the end and the beginning of the pressure gradient is maximum ${ }^{28}$. For instance, Figure 7 shows the physical states of carbon dioxide at the beginning (blue color) and at the end (green color) of an ABPR gradient (1500 to $3500 \mathrm{psi}$ ) along the column when it is placed under adiabatic conditions (the enthalpy of carbon dioxide remains constant) for an inlet temperature fixed at $90^{\circ} \mathrm{C}$. The temperature change at the column outlet is $80-53=27^{\circ} \mathrm{C}$, which is very large. Potentially, a reduction of this temperature difference could be expected if the column was placed in an oven compartment heated at $90^{\circ} \mathrm{C}$. It is expected the outlet temperature to be around $80^{\circ} \mathrm{C}$ (instead of $53^{\circ} \mathrm{C}$ ) and $87^{\circ} \mathrm{C}$ (instead of $80^{\circ} \mathrm{C}$ ) before and after the $\mathrm{ABPR}$ gradient, respectively. In the end, the temperature difference would drop from $27^{\circ} \mathrm{C}$ to only $7^{\circ} \mathrm{C}$. Figure 8 provides two experimental chromatograms 
that confirm this suggested scenario: in blue color, the column is run under adiabatic conditions; in black color, standard oven conditions are applied under atmospheric pressure $\left(90^{\circ} \mathrm{C}\right)$. It is remarkable that the relative performance between these two thermal column configurations depends on whether isobaric or ABPR gradient conditions are applied. Under isobaric conditions (e.g., during a steady-state temperature regime), insulating the column is always the best option as it was previously observed for other sample mixtures: the steepness of the radial density gradients are always kept to a minimum. In contrast, the opposite trend is observed under ABPR gradient conditions : the temperature difference between the two steady state regimes is minimized when the column is placed in the heated oven compartment and the peak resolution is improving despite some residual heat loss at the column wall. It is the best compromise found under these ABPR gradient conditions. 


\section{Conclusion}

This work demonstrated that it is possible to use low-density mobile phases (such as carbon dioxide at elevated temperatures and at low pressures) for the fast (short $3.0 \mathrm{~mm} \times 150 \mathrm{~mm}$ columns) and high-resolution (sub-2 $\mu \mathrm{m}$ particles were used) separation of volatile compounds. The most critical implementation of LDFC is the application of a high-vacuum $\left(<10^{-4}\right.$ Torr $)$ around the column in order to eliminate nefarious thermal effects and to preserve the integrity of the column efficiency. Unlike classical capillary GC $\times$ LC, capillary GC $\times$ SFC, or capillary SFC-FID analytical methods, LDFC combined with vacuum technology can be used with short $3.0 \mathrm{~mm}$ i.d. analytical columns for the rapid and high-resolution separation of both volatile and non-volatile compounds in a single run. For instance, it can take less than a minute to fully resolve volatile $n$-alkanes from $\mathrm{C}_{5}$ to $\mathrm{C}_{12}$ under isobaric conditions and less than 5 five min to baseline separate non-volatile $n$-alkanes from $\mathrm{C}_{20}$ to $\mathrm{C}_{40}$ under $\mathrm{ABPR}$ pressure gradient conditions. For the sake of comparison, traditional capillary SFC-FID methods cannot analyze the most volatile alkanes $\left(<\mathrm{C}_{10}\right)$ because the capillary columns are not retentive enough and the analysis time can be significantly larger than 10 min.

The present work also raised a fundamental issue which is related to the application and performance of ABPR pressure gradients, which induce a significant change in the temperature of low-density mobile phase along the column and some heat exchanges between the mobile phase and the stainless steel wall of the columns. The practical goal is to minimize this heat flux by minimizing the temperature variation of the mobile phase from the beginning to the end of the gradient. A compromise has eventually to be found between the application of a high-vacuum and wall temperature-control in an oven compartment. Heated air at intermediate pressures could be the best compromise in terms of column efficiency when running ABPR gradients. 


\section{${ }_{412} \quad 5 \quad$ Acknowledgements}

${ }_{413}$ The authors would like to acknowledge and thank Abhijit Tarafder (Waters, Milford, MA, USA) for

414 the supply of the isenthalpic plots from the NIST REFPROP data and Shawn Hellmueller (Waters,

415 Milford, MA, USA) for providing us with the plant extract (terpenes and cannabinoids). 


\section{References}

[1] B. Kolb, L. Ettre, Static Headspace-Gas Chromatography, Theory and Practice, 2nd Edition, John Wiley \& Sons, Inc., 2006.

[2] L. Snyder, High Performance Liquid Chromatography - Advances and Perspectives, Elsevier, Amsterdam, 1986.

[3] D. Thiebault, Separations of petroleum products involving supercritical fluid chromatography, J. Chromatogr. A 1252 (2012) 177-188.

[4] J. Giddings, Dynamics of Chromatography, Marcel Dekker, New York, NY, 1965.

[5] P. Myers, K. Bartle, T. Edge, Unified chromatography: How the history of sfc brings separation methods together, Chromatography Today August/September (2014) 30-32.

[6] G. Guiochon, A. Tarafder, Fundamental challenges and opportunities for preparative supercritical fluid chromatography, J. Chromatogr. A 1218 (2011) 1037-1114.

[7] T. A. Berger, B. K. Berger, K. Fogleman, Chromatographic separations and analysis: Supercritical fluid chromatography for chiral analysis and semi-preparative purification, Reference Module in Chemistry, Molecular Sciences and Chemical Engineering, from Comprehensive Chirality 8 (2014) 354-392.

[8] T. Berger, Packed Column SFC, 1st ed., Royal Society of Chemistry, London, UK, 1995.

[9] M. McNally, Supercritical fluid chromatography, Comprehensive Analytical Chemistry 47 (2006) 561-574.

[10] E. Leselier, Retention mechanisms in super/subcritical fluid chromatography on packed columns, J. Chromatogr. A 1216 (2009) 1881-1890.

[11] A. Tarafder, G. Guiochon, Use of isopycnic plots in designing operations of supercritical fluid chromatography: II. the isopycnic plots and the selection of the operating pressuretemperature zone in supercritical fluid chromatography, J. Chromatogr. A 1218 (2011) 4576-4585. 
[12] M. Enmark, D. Asberg, H. Leek, K. Ohlen, M. Klarqvist, J. Samuelsson, T. Fornstedt, Evaluation of scale-up from analytical to preparative supercritical fluid chromatography, J. Chromatogr. A 1425 (2015) 280-286.

[13] H.-J. Lin, C. Horvath, Viscous dissipation in packed beds, Chem. Eng. Sci. 36 (1981) 47-55.

[14] H. Poppe, J. Kraak, J. Huber, Temperature gradients in hplc columns due to viscous dissipation, Chromatographia 14 (1981) 515-523.

[15] H. Poppe, J. Kraak, Influence of thermal conditions on the efficiency of high-performance liquid chromatographic columns, J. Chromatogr. 282 (1983) 515-523.

[16] M. Martin, G. Guiochon, Effect of high pressure in liquid chromatography, J. Chromatogr. A 1090 (2005) 16-38.

[17] F. Gritti, M. Martin, G. Guiochon, Influence of viscous friction heating on the efficiency of columns operated under very high pressures, Anal. Chem. 81 (2009) 3365-3384.

[18] F. Gritti, G. Guiochon, Complete temperature profiles in ultra-high- pressure liquid chromatography columns, Anal. Chem. 80 (2008) 5009-5020.

[19] F. Gritti, G. Guiochon, Optimization of the thermal environment of columns packed with very fine particles, J. Chromatogr. A 1216 (2009) 1353-1362.

[20] D. Poe, J. Schroden, Effects of pressure drop, particle size and thermal conditions on retention and efficiency in supercritical fluid chromatography, J. Chromatogr. A 1216 (2009) 7915-7926.

[21] K. Kaczmarski, D. Poe, G. Guiochon, Numerical modeling of the elution peak profiles of retained solutes in supercritical fluid chromatography, J. Chromatogr. A 1217 (2010) 6578.

[22] K. Kaczmarski, D. Poe, A. Tarafder, G. Guiochon, Efficiency of supercritical fluid chromatography columns in different thermal environments, J. Chromatogr. A 1291 (2013) 155-173.

[23] K. Kaczmarski, D. Poe, A. Tarafder, G. Guiochon, Efficiency of supercritical fluid chromatography columns in different thermal environments, J. Chromatogr. A 1291 (2013) 155-173. 
[24] F. Gritti, M. Gilar, J. A. Jarrell, Achieving quasi-adiabatic thermal environment to maximize resolution power in very high-pressure liquid chromatography : Theory, models, and experiments, J. Chromatogr. A 1444 (2016) 86-98.

[25] F. Gritti, M. Gilar, J. A. Jarrell, Quasi-adiabatic vacuum-based column housing for very highpressure liquid chromatography, J. Chromatogr. A 1456 (2016) 226-234.

[26] F. Gritti, M. Fogwill, M. Gilar, J. A. Jarrell, Maximizing performance in supercritical fluid chromatography using low-density mobile phase, J. Chromatogr. A submitted for publication.

[27] F. Gritti, Anomalies in retention and column efficiencies in supercritical fluid chromatography : interpretation from the analyte-analyte solvent competitive adsorption, J. Chromatogr. A 1468 (2016) 209-216.

[28] A. Tarafder, P. Iraneta, G. Guiochon, K. Kaczmarski, D. P. Poe, Estimations of temperature deviations in chromatographic columns using isenthalpic plots. i. theory for isocratic systems, J. Chromatogr. A 1366 (2014) 126-135. 


\section{Figure captions}

1 Isobaric separation of three terpenes and one cannabidiol product extracted (spherex CBD) from marijuana plants. Terpenes are the most volatile and first eluted compounds as indicated in the bottom graph. The cannabindiol compound is trapped at the column inlet and is not detected. Carbon disulfide is the sample solvent. See the detailed experimental conditions (flow rate, inlet temperature of carbon dioxide, and ABPR pressure) in the experimental section 2.4.1. The column is fully embedded inside the $6 \mathrm{~cm}$ i.d. vacuum tube placed horizontally under still-air conditions at $24^{\circ} \mathrm{C}$. FID detection. (Top graph) The housing air pressure is atmospheric pressure and the analysis time is $0.95 \mathrm{~min}$. (Bottom graph) The housing air pressure is $10^{-5}$ Torr and the analysis time is $1.5 \mathrm{~min} . \ldots \ldots \ldots \ldots$

2 Same isobaric separation of terpenes as in Figure 1, except the inlet temperature of carbon dioxide is $90^{\circ} \mathrm{C}$ and the thermal environment of the column is changed. FID detection (Top graph) The column is placed in the conventional oven compartment of the ACQUITY UPC ${ }^{2}$ system. (Bottom graph) The column is fully embedded inside the $6 \mathrm{~cm}$ i.d. vacuum tube placed horizontally under still-air conditions at $24^{\circ} \mathrm{C}$, the housing air pressure is $10^{-5}$ Torr. In both cases, the analysis time is 0.7

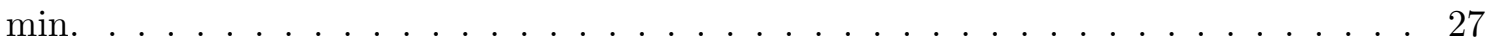

3 Isobaric separation of eleven volatile $n$-alkanes and 2,2,4-trimethylpentane. Those alkanes are the most volatile fraction of petroleum products. See the detailed experimental conditions (flow rate, inlet temperature of carbon dioxide, and ABPR pressure) in the experimental section 2.4.2. The column is fully embedded inside the $6 \mathrm{~cm}$ i.d. vacuum tube placed horizontally under still-air conditions at $24^{\circ} \mathrm{C}$. FID detection. (Top graph) The housing air pressure is atmospheric pressure and the analysis time is 0.75 min. (Bottom graph) The housing air pressure is $10^{-5}$ Torr and the analysis time is 1.4 min. . . . . . . . . . . . . . . . 28 
4 Isobaric analysis of alkanes present in gasoline Gulf 87 containing at least $10 \%$ ethanol in volume (see the retained overloaded band profile of ethanol in the right side of both chromatograms). See the detailed experimental conditions (flow rate, inlet temperature of carbon dioxide, and ABPR pressure) in the experimental section 2.4.3. The column is fully embedded inside the $6 \mathrm{~cm}$ i.d. vacuum tube placed horizontally under still-air conditions at $24^{\circ} \mathrm{C}$. FID detection. (Top graph) The housing air pressure is atmospheric pressure and the elution time of the front shock of the ethanol band is $13.5 \mathrm{~min}$. (Bottom graph) The housing air pressure is $10^{-5}$ Torr and the elution time of the front shock of the ethanol band decreases to only 8.7 min. . . . . . . . . . . . . . . . . . . . . 29

5 Same isobaric separation of gasoline as in Figure 1, except the inlet temperature of carbon dioxide is $90^{\circ} \mathrm{C}$ and the thermal environment of the column is changed. FID detection. (Top graph) The column is placed in the conventional oven compartment of the ACQUITY UPC ${ }^{2}$ system and the elution time of the front shock of the ethanol band is $9.5 \mathrm{~min}$. (Bottom graph) The column is fully embedded inside the $6 \mathrm{~cm}$ i.d. vacuum tube placed horizontally under still-air conditions at $24^{\circ} \mathrm{C}$ and the housing air pressure is $10^{-5}$ Torr and the elution time of the front shock of the ethanol band

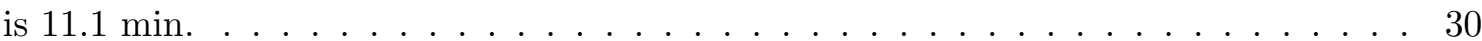

6 Isobaric followed by an $\mathrm{ABPR}$ pressure gradient analysis of light alkanes $\left(\mathrm{C}_{5}\right.$ to $\left.\mathrm{C}_{20}\right)$ mixed with a paraffin wax $\left(\mathrm{C}_{20}\right.$ to $\left.\mathrm{C}_{40}\right)$. See the detailed experimental conditions (flow rate, inlet temperature of carbon dioxide, and ABPR pressure) in the experimental section 2.4.4. The column is fully embedded inside the $6 \mathrm{~cm}$ i.d. vacuum tube placed horizontally under still-air conditions at $24^{\circ} \mathrm{C}$. FID detection. (Top graph) The housing air pressure is atmospheric pressure. (Bottom graph) The housing air pressure is $10^{-5}$ Torr. In both cases, the total analysis time is $5.20 \mathrm{~min}$. . . . . . . . 31 
7 Illustration of the change in the temperature profile of the mobile phase (carbon dioxide) along the column before (solid blue line) and after (solid green line) an ABPR pressure gradient is applied. The outlet column pressure is increased from $1500 \mathrm{psi}$ (blue line) to $3500 \mathrm{psi}$ (green line). The inlet temperature is fixed at 90 ${ }^{o} \mathrm{C}$. The column is placed under adiabatic conditions. Note the significant increase of the eluent temperature at the column outlet from $53{ }^{\circ} \mathrm{C}$ to $80{ }^{\circ} \mathrm{C}$. The solid red lines represent isenthalpic (or constant enthalpy in $\mathrm{J} / \mathrm{g}$ ) lines. . . . . . . . . . . . . 32

8 Same isobaric followed by an ABPR pressure gradient separation of alkanes $\left(\mathrm{C}_{5}\right.$ to $\left.\mathrm{C}_{20}\right)$ mixed with a paraffin wax $\left(\mathrm{C}_{20}\right.$ to $\left.\mathrm{C}_{40}\right)$ as in Figure 7 , except the inlet temperature of carbon dioxide is $65^{\circ} \mathrm{C}$ and the thermal environment of the column is changed. The FID signal shown in black color corresponds to the configuration where the column is placed in the conventional oven compartment of the ACQUITY $\mathrm{UPC}^{2}$ system. The solid blue line is the recorded chromatogram when the column is fully embedded inside the $6 \mathrm{~cm}$ i.d. vacuum tube placed horizontally under stillair conditions at $24^{\circ} \mathrm{C}$ and when the housing air pressure is set at $10^{-5}$ Torr. The analysis time is 7.5 min. . . . . . . . . . . . . . . . . 33 
Figure 1: Isobaric separation of three terpenes and one cannabidiol product extracted (spherex CBD) from marijuana plants. Terpenes are the most volatile and first eluted compounds as indicated in the bottom graph. The cannabindiol compound is trapped at the column inlet and is not detected. Carbon disulfide is the sample solvent. See the detailed experimental conditions (flow rate, inlet temperature of carbon dioxide, and ABPR pressure) in the experimental section 2.4.1. The column is fully embedded inside the $6 \mathrm{~cm}$ i.d. vacuum tube placed horizontally under still-air conditions at $24^{\circ} \mathrm{C}$. FID detection. (Top graph) The housing air pressure is atmospheric pressure and the analysis time is $0.95 \mathrm{~min}$. (Bottom graph) The housing air pressure is $10^{-5}$ Torr and the analysis time is $1.5 \mathrm{~min}$.
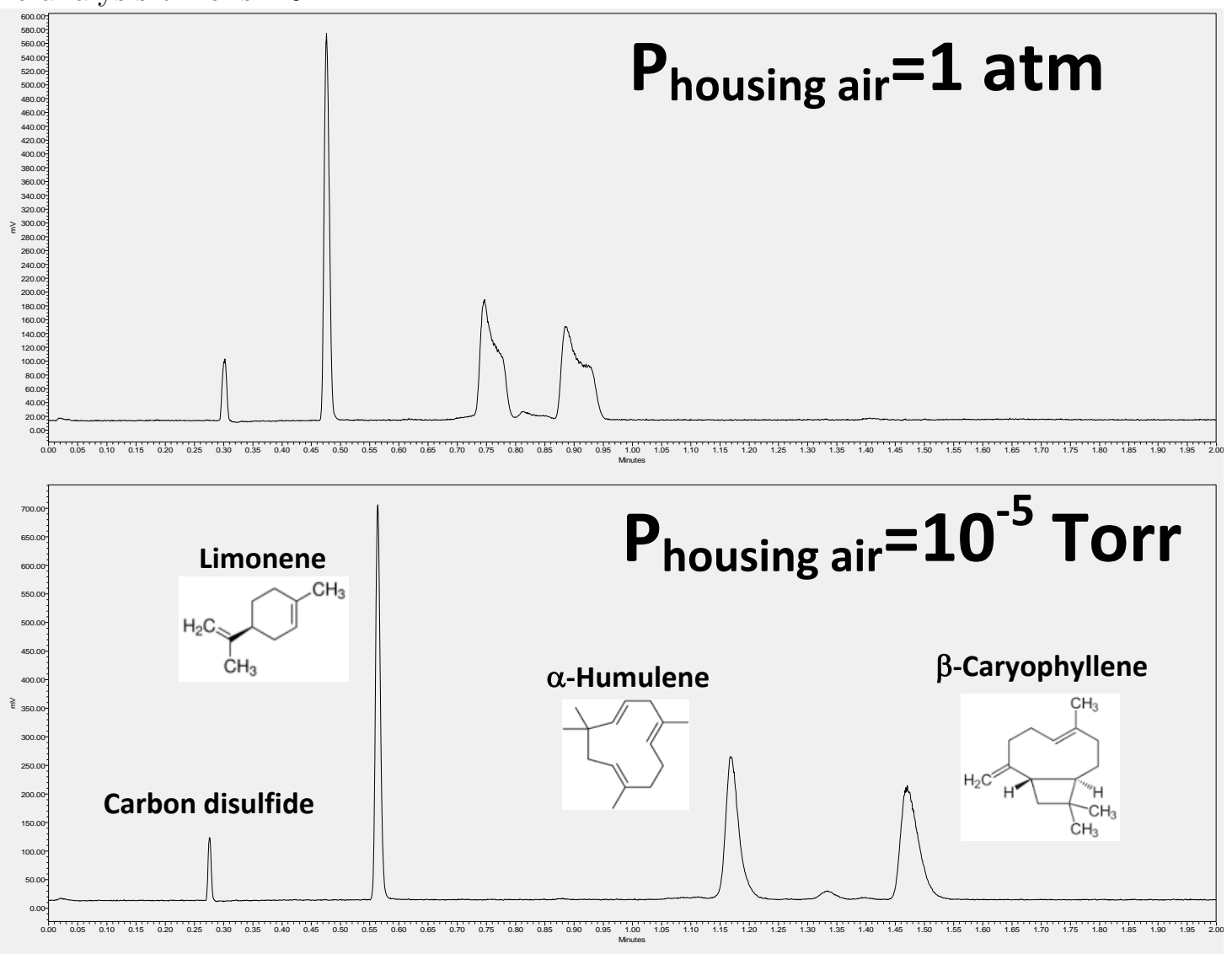
Figure 2: Same isobaric separation of terpenes as in Figure 1, except the inlet temperature of carbon dioxide is $90^{\circ} \mathrm{C}$ and the thermal environment of the column is changed. FID detection (Top graph) The column is placed in the conventional oven compartment of the ACQUITY UPC ${ }^{2}$ system. (Bottom graph) The column is fully embedded inside the $6 \mathrm{~cm}$ i.d. vacuum tube placed horizontally under still-air conditions at $24^{\circ} \mathrm{C}$, the housing air pressure is $10^{-5}$ Torr. In both cases, the analysis time is $0.7 \mathrm{~min}$.
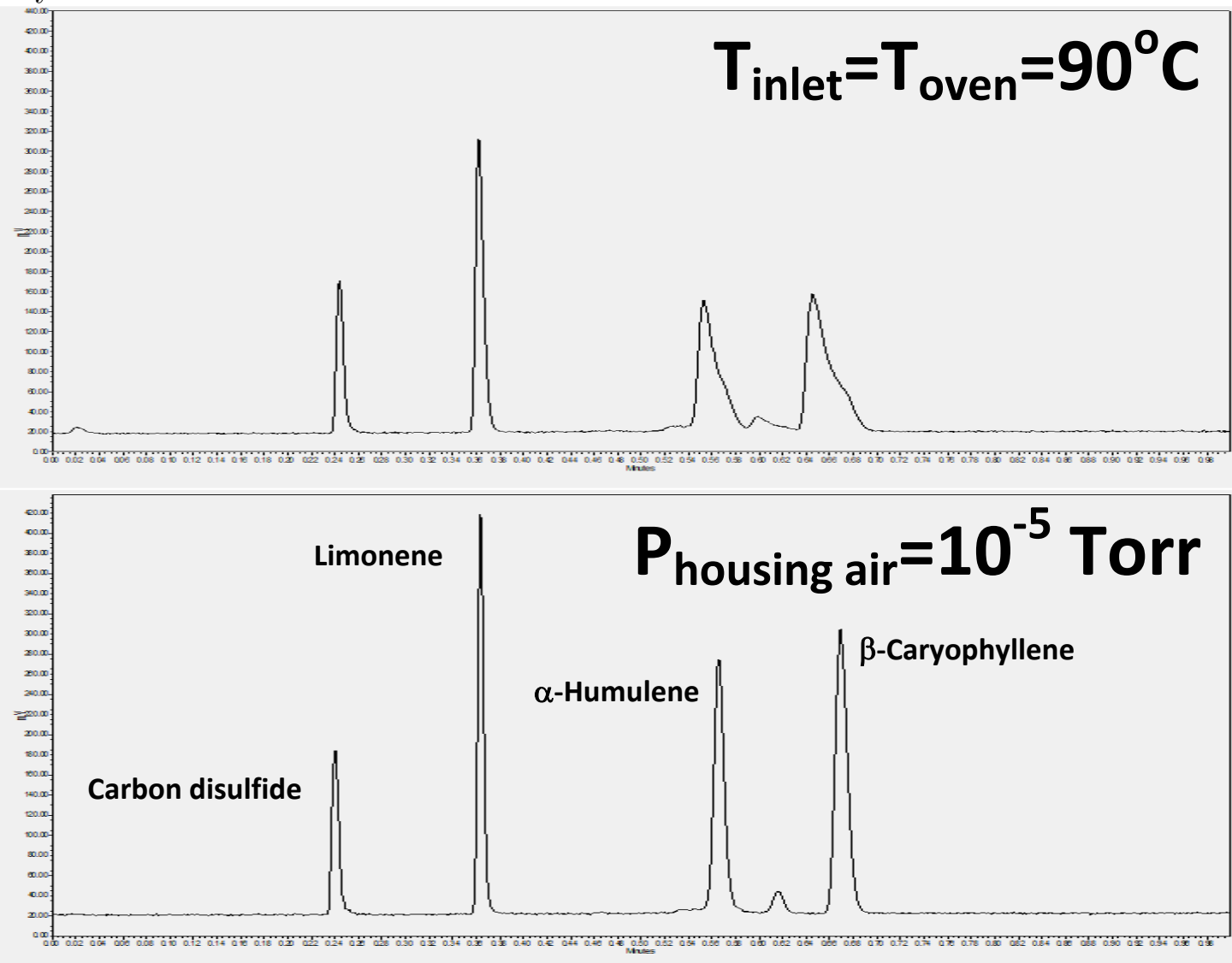
Figure 3: Isobaric separation of eleven volatile $n$-alkanes and 2,2,4-trimethylpentane. Those alkanes are the most volatile fraction of petroleum products. See the detailed experimental conditions (flow rate, inlet temperature of carbon dioxide, and ABPR pressure) in the experimental section 2.4.2. The column is fully embedded inside the $6 \mathrm{~cm}$ i.d. vacuum tube placed horizontally under still-air conditions at $24^{\circ} \mathrm{C}$. FID detection. (Top graph) The housing air pressure is atmospheric pressure and the analysis time is $0.75 \mathrm{~min}$. (Bottom graph) The housing air pressure is $10^{-5}$ Torr and the analysis time is $1.4 \mathrm{~min}$.
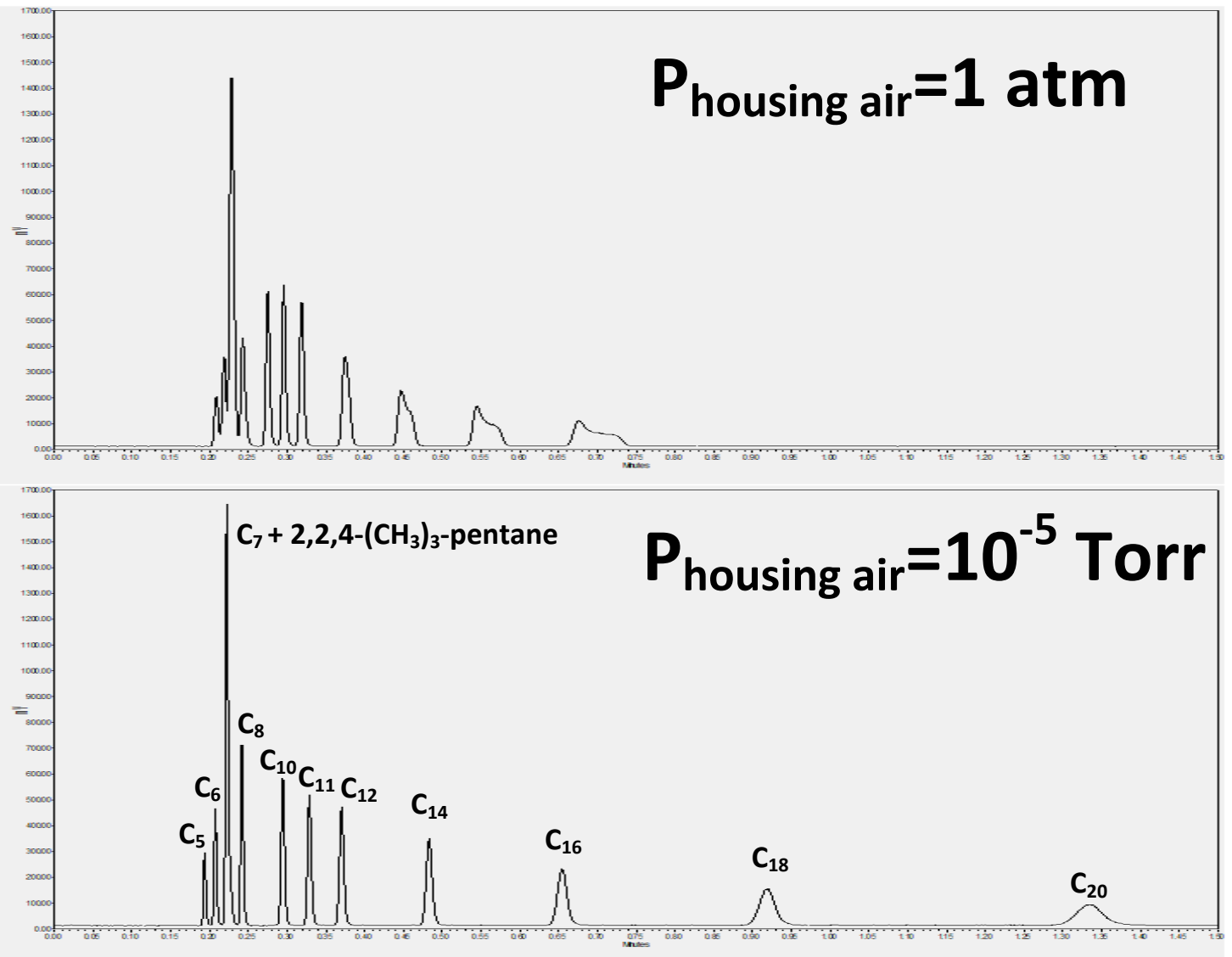
Figure 4: Isobaric analysis of alkanes present in gasoline Gulf 87 containing at least 10\% ethanol in volume (see the retained overloaded band profile of ethanol in the right side of both chromatograms). See the detailed experimental conditions (flow rate, inlet temperature of carbon dioxide, and ABPR pressure) in the experimental section 2.4.3. The column is fully embedded inside the $6 \mathrm{~cm}$ i.d. vacuum tube placed horizontally under still-air conditions at $24^{\circ} \mathrm{C}$. FID detection. (Top graph) The housing air pressure is atmospheric pressure and the elution time of the front shock of the ethanol band is $13.5 \mathrm{~min}$. (Bottom graph) The housing air pressure is $10^{-5}$ Torr and the elution time of the front shock of the ethanol band decreases to only $8.7 \mathrm{~min}$.
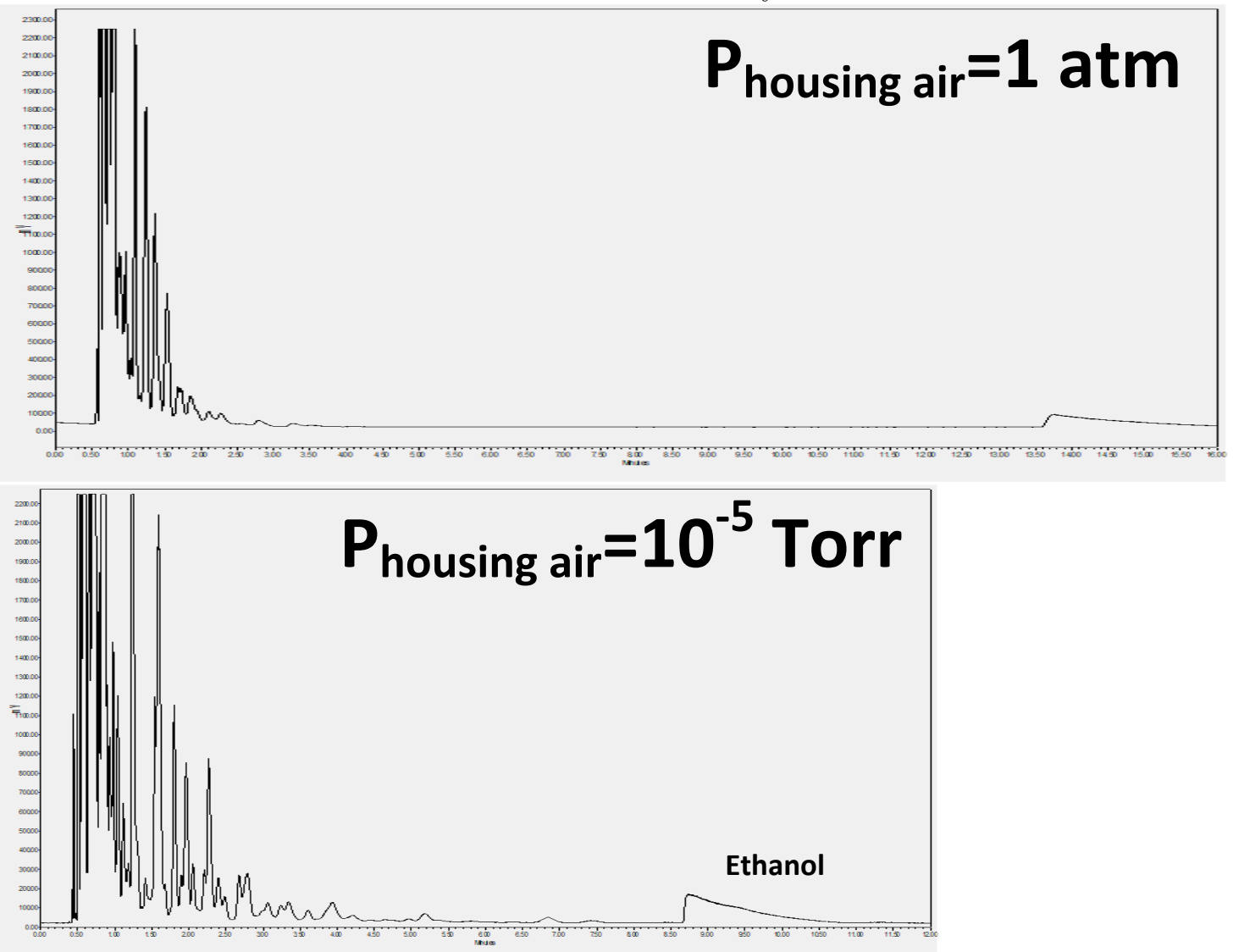
Figure 5: Same isobaric separation of gasoline as in Figure 1, except the inlet temperature of carbon dioxide is $90^{\circ} \mathrm{C}$ and the thermal environment of the column is changed. FID detection. (Top graph) The column is placed in the conventional oven compartment of the ACQUITY UPC ${ }^{2}$ system and the elution time of the front shock of the ethanol band is $9.5 \mathrm{~min}$. (Bottom graph) The column is fully embedded inside the $6 \mathrm{~cm}$ i.d. vacuum tube placed horizontally under still-air conditions at $24^{\circ} \mathrm{C}$ and the housing air pressure is $10^{-5}$ Torr and the elution time of the front shock of the ethanol band is $11.1 \mathrm{~min}$.
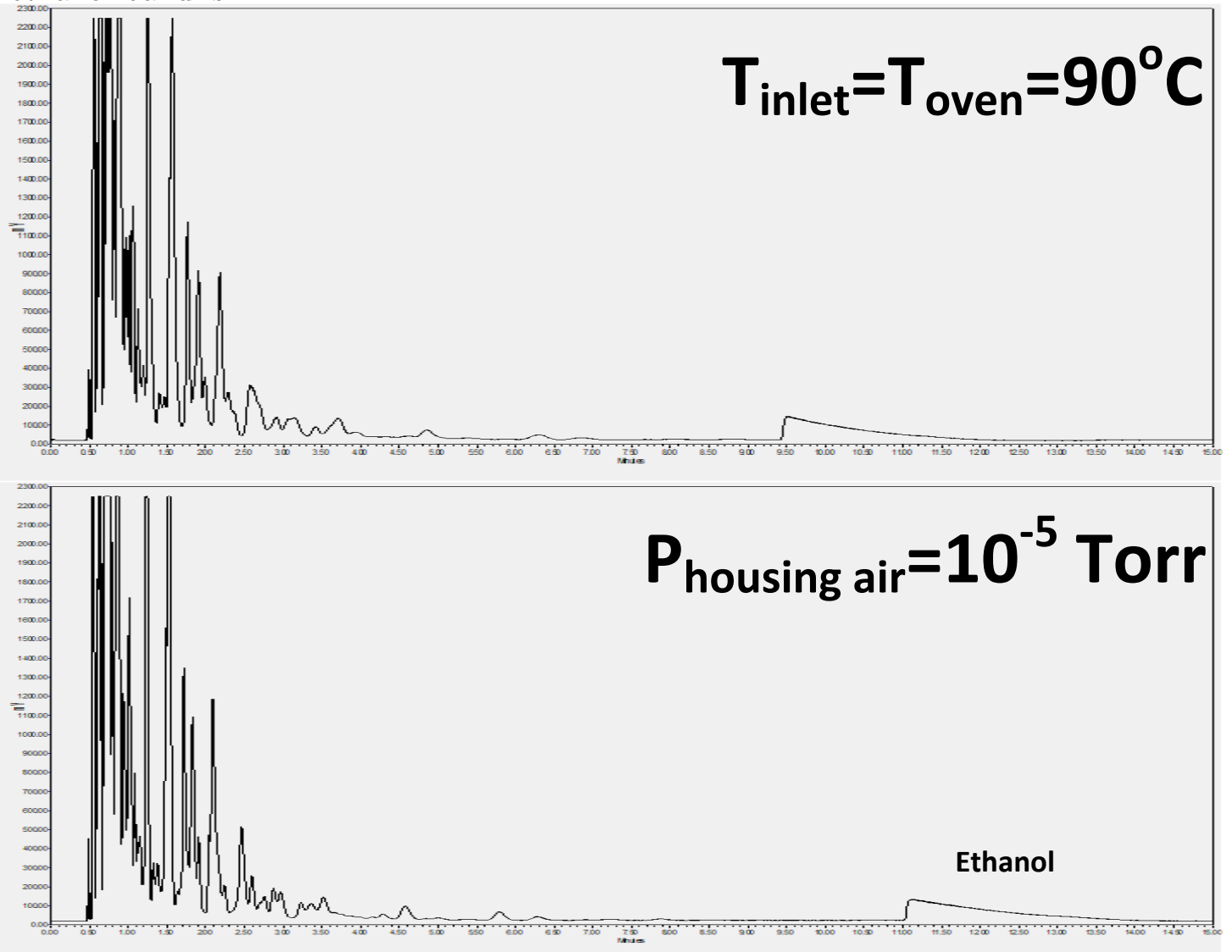
Figure 6: Isobaric followed by an $\mathrm{ABPR}$ pressure gradient analysis of light alkanes $\left(\mathrm{C}_{5}\right.$ to $\left.\mathrm{C}_{20}\right)$ mixed with a paraffin wax $\left(\mathrm{C}_{20}\right.$ to $\left.\mathrm{C}_{40}\right)$. See the detailed experimental conditions (flow rate, inlet temperature of carbon dioxide, and ABPR pressure) in the experimental section 2.4.4. The column is fully embedded inside the $6 \mathrm{~cm}$ i.d. vacuum tube placed horizontally under still-air conditions at $24^{\circ} \mathrm{C}$. FID detection. (Top graph) The housing air pressure is atmospheric pressure. (Bottom graph) The housing air pressure is $10^{-5}$ Torr. In both cases, the total analysis time is 5.20 min.
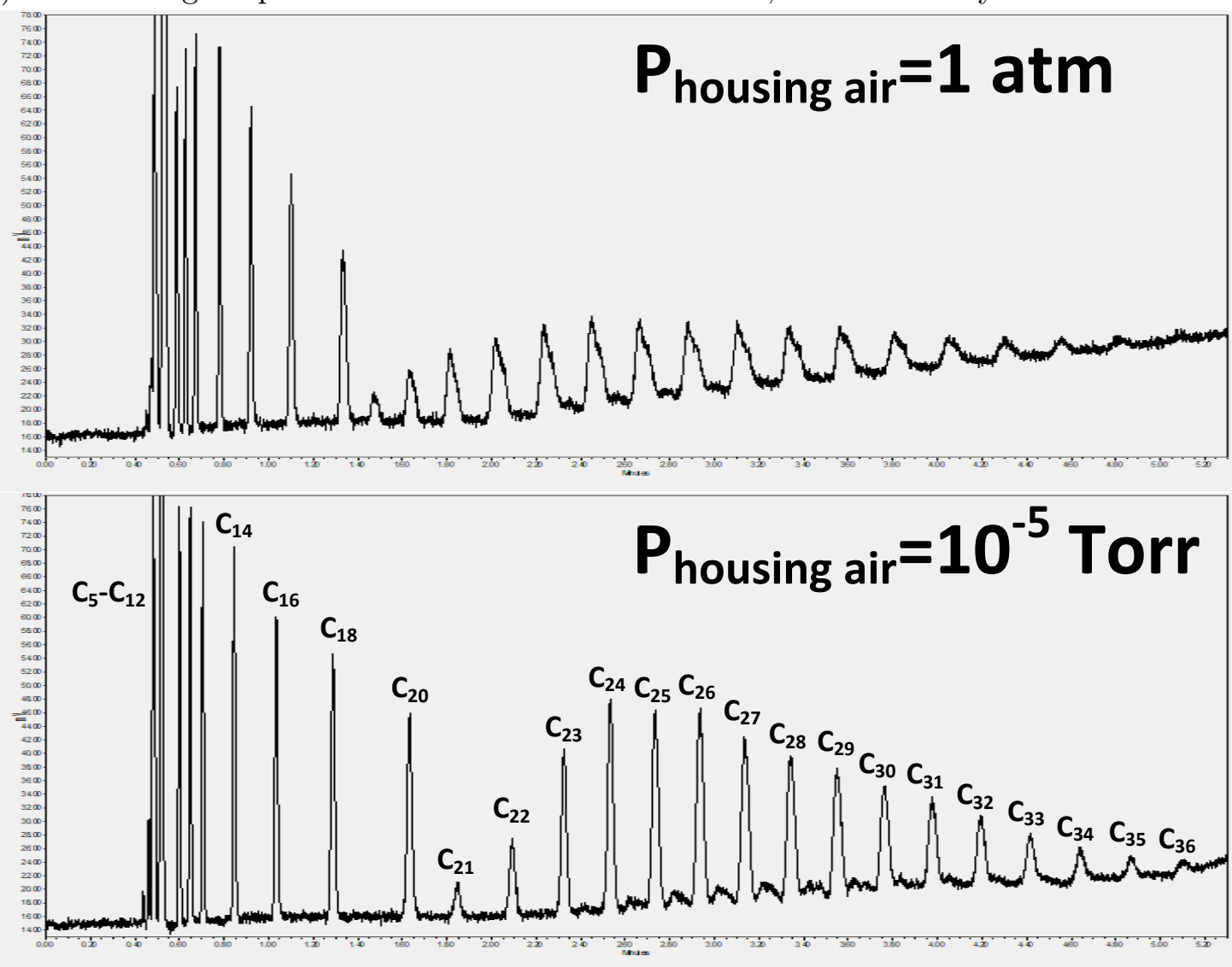
Figure 7: Illustration of the change in the temperature profile of the mobile phase (carbon dioxide) along the column before (solid blue line) and after (solid green line) an ABPR pressure gradient is applied. The outlet column pressure is increased from 1500 psi (blue line) to 3500 psi (green line). The inlet temperature is fixed at $90{ }^{\circ} \mathrm{C}$. The column is placed under adiabatic conditions. Note the significant increase of the eluent temperature at the column outlet from $53{ }^{\circ} \mathrm{C}$ to $80{ }^{\circ} \mathrm{C}$. The solid red lines represent isenthalpic (or constant enthalpy in $\mathrm{J} / \mathrm{g}$ ) lines.

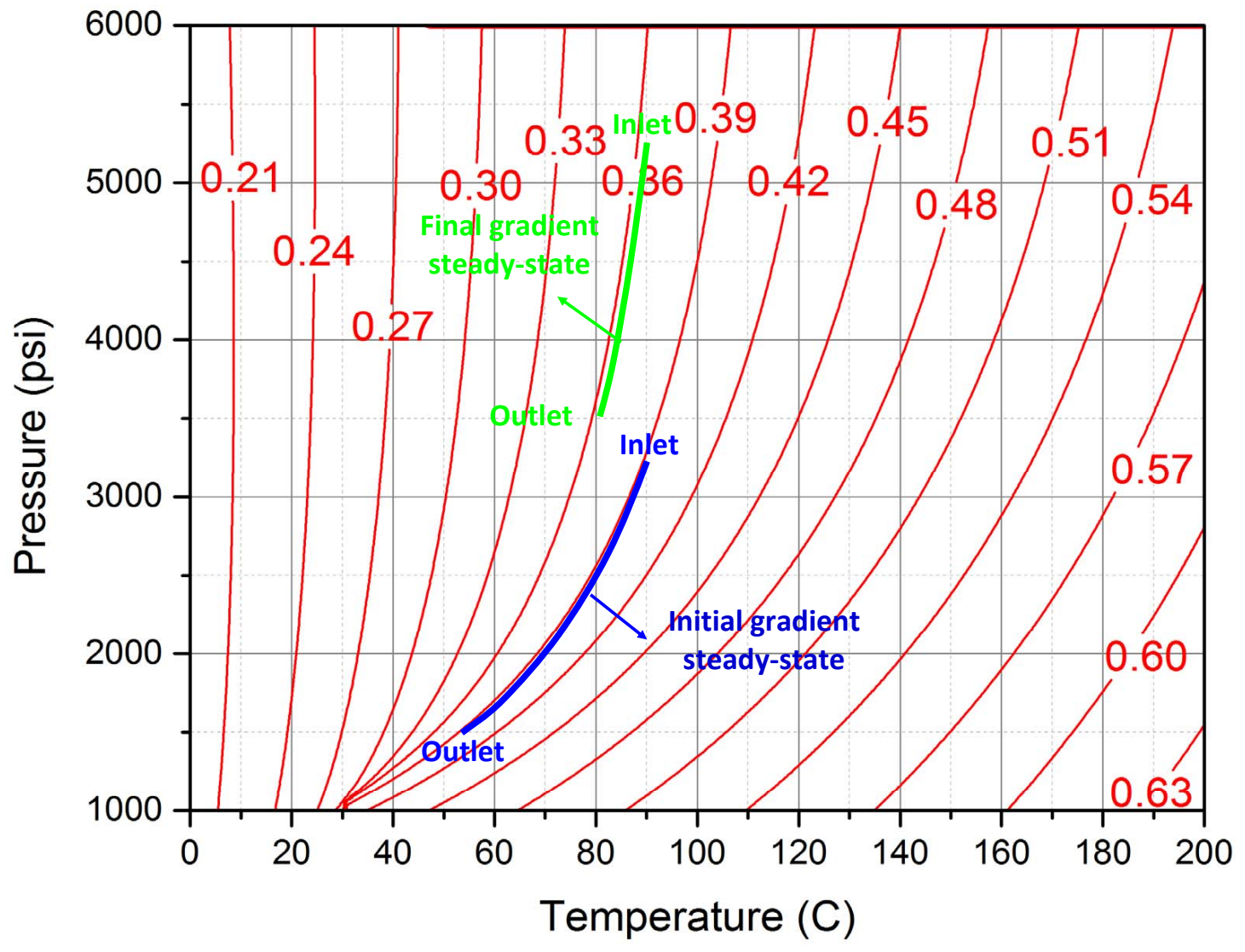


Figure 8: Same isobaric followed by an ABPR pressure gradient separation of alkanes $\left(\mathrm{C}_{5}\right.$ to $\left.\mathrm{C}_{20}\right)$ mixed with a paraffin wax $\left(\mathrm{C}_{20}\right.$ to $\left.\mathrm{C}_{40}\right)$ as in Figure 7 , except the inlet temperature of carbon dioxide is $65^{\circ} \mathrm{C}$ and the thermal environment of the column is changed. The FID signal shown in black color corresponds to the configuration where the column is placed in the conventional oven compartment of the ACQUITY UPC ${ }^{2}$ system. The solid blue line is the recorded chromatogram when the column is fully embedded inside the $6 \mathrm{~cm}$ i.d. vacuum tube placed horizontally under still-air conditions at $24^{\circ} \mathrm{C}$ and when the housing air pressure is set at $10^{-5}$ Torr. The analysis time is $7.5 \mathrm{~min}$.

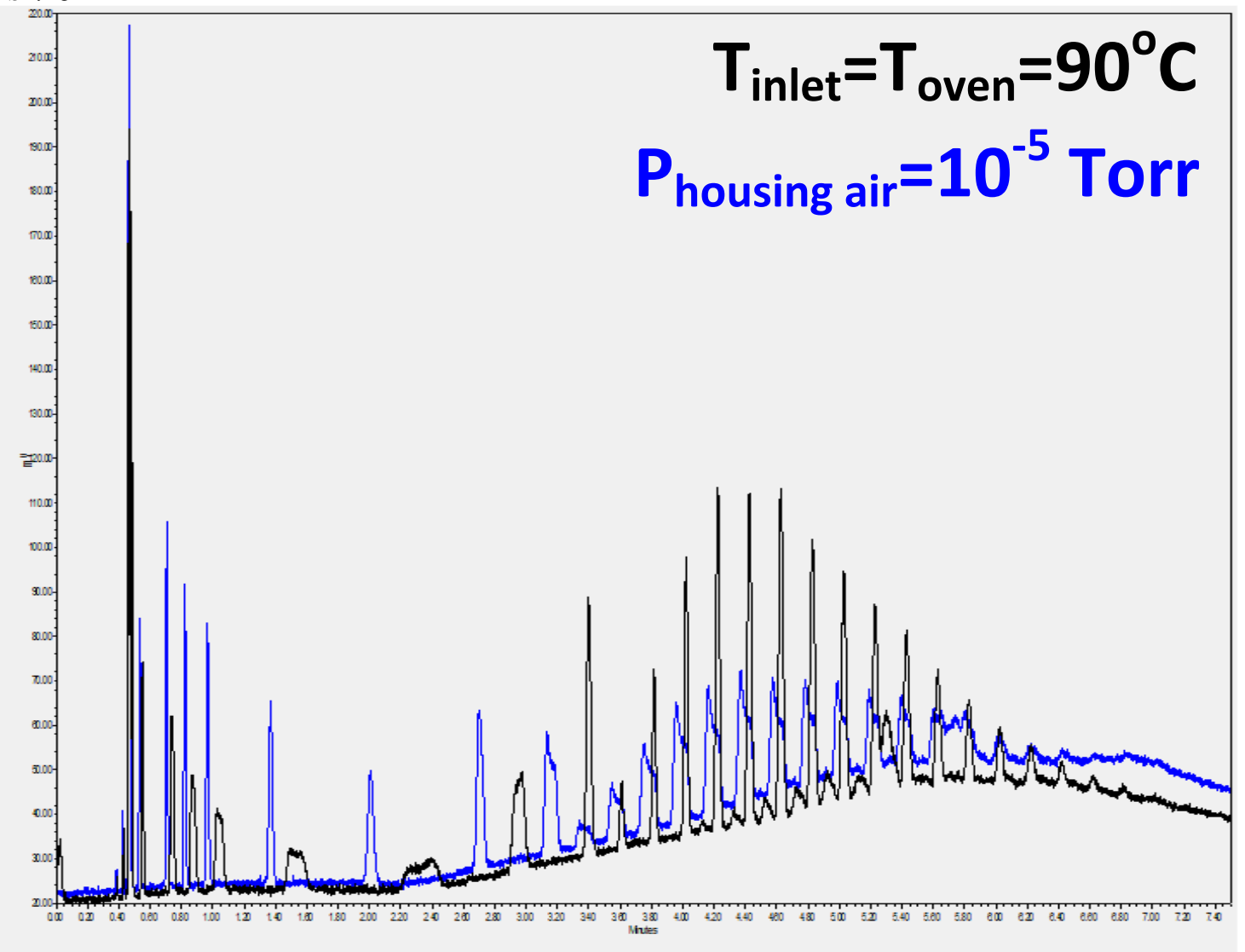

